\title{
Diffraction of plane SH waves by a semi-circular cavity in half-space*
}

\author{
Jianwen Liang ${ }^{1, *}$ Hao Luo $^{2}$ and Vincent W Lee ${ }^{3}$ \\ ${ }^{1}$ Department of Civil Engineering, Tianjin University, Tianjin 300072, China \\ ${ }^{2}$ HNTB Corporation, Santa Ana, CA 92707, USA \\ ${ }^{3}$ University of Southern California, Los Angeles, CA 90089-2531, USA
}

\begin{abstract}
This paper presents a closed-form solution for diffraction of plane SH waves by a semi-circular cavity in half-space by using wave function expansion method. Accuracy of the solution is checked by the displacement residual and stress residual along the boundaries. Numerical results show that there are notable differences for response amplitudes between a semi-circular cavity and a whole-circular cavity in a half-space.
\end{abstract}

Key words: diffraction; SH wave; semi-circular cavity; wave function expansion method; closed-form solution CLC number: P315.3 $3^{+}$Document code: A

\section{Introduction}

Diffraction of elastic waves around underground cavities can be studied by analytical methods or numerical methods. Although numerical methods can be used for cavities of arbitrary shape, analytical methods are essential for exploring the physical nature of the problems, and for checking the accuracy of numerical methods. Pao and Mow (1973) presented an exact analytical solution for dynamic stress concentration of circular cavity in whole space for incident elastic waves. Lee (1977) and Lee and Trifunac (1979) extended the solution to half-space by the image method, and studied the diffraction of $\mathrm{SH}$ waves by a circular cavity and lined tunnel. Liu and Lin (2003) studied the diffraction of plane SH waves by a circular cavity by complex function method.

In recent years, auxiliary function method was proposed by Yuan and Men (1992) and Yuan and Liao (1996) to solve the problem of diffraction of SH waves by a cylindrical hill. And the method was used to develop an analytical solution for diffraction of $\mathrm{SH}$ waves by a semi-circular hill with an inside semi-circular tunnel (Lee et al, 2004).

\footnotetext{
* Received 7 November 2009; accepted in revised form 1 January 2010; published 10 February 2010.

* Corresponding author. e-mail: liang@tju.edu.cn

(C) The Seismological Society of China and Springer-Verlag Berlin Heidelberg 2010
}

The purpose of this study is to present an exact analytical solution for diffraction of plane $\mathrm{SH}$ waves by a semi-circular cavity in half-space by using auxiliary function method, and to explore the difference of wave diffraction around a semi-circular cavity in half-space and wave diffraction around a whole-circular cavity in half-space.

\section{Formulation}

The two-dimensional plane strain model is shown in Figure 1. A semi-circular cavity with radius $a$ is buried in an elastic, isotropic and homogeneous half-space. The depth of the cavity is denoted by $d$. The free surface of the half-space, upper semi-circular boundary of the cavity and lower flat boundary of the cavity are denoted by $\Gamma, L_{1}$ and $L_{2}$, respectively.

The semi-circular cavity is excited by plane $\mathrm{SH}$ wave $u^{(i)}$, with incident angle $\alpha$, shear wave velocity $c_{\beta}$, circular frequency $\omega$, and amplitude $u_{0}$. The incident wave is reflected and diffracted, and the total wave $u$ in the half-space satisfies the differential equation

$$
\frac{\partial^{2} u}{\partial r_{1}^{2}}+\frac{1}{r_{1}} \frac{\partial u}{\partial r_{1}}+\frac{1}{r_{1}^{2}} \frac{\partial^{2} u}{\partial \theta_{1}^{2}}=\frac{1}{c_{\beta}^{2}} \frac{\partial^{2} u}{\partial t^{2}} .
$$

The boundary conditions of the problem are the traction-free boundary conditions along the boundaries $\Gamma, L_{1}$ and $L_{2}$, respectively, which can be expressed as 

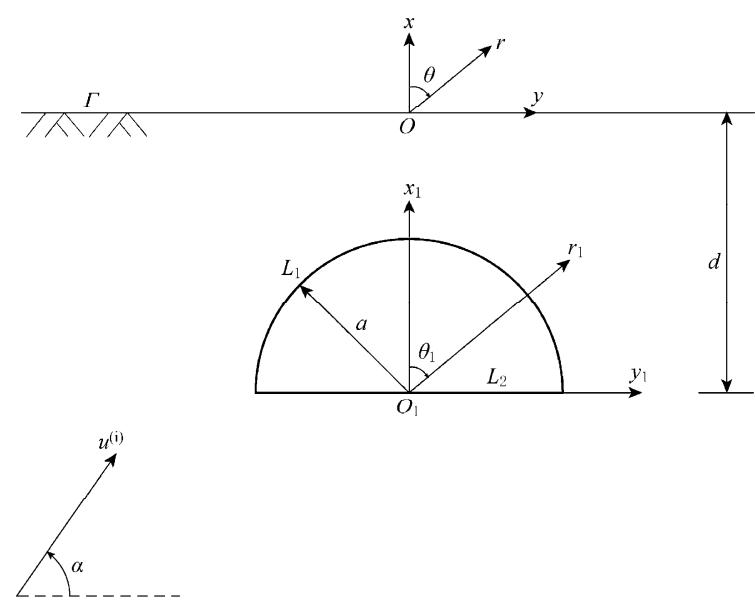

Figure 1 Semi-circular cavity in half-space.

$$
\begin{array}{ll}
\left.\sigma_{\theta z}\right|_{\theta= \pm \frac{\pi}{2}}=0 & (r, \theta) \in \Gamma, \\
\left.\sigma_{r_{1} z}\right|_{r_{1}=a}=0 & \left(r_{1}, \theta_{1}\right) \in L_{1},
\end{array}
$$

and

$$
\left.\sigma_{\theta_{1} z}\right|_{\theta_{1}= \pm \frac{\pi}{2}}=0 \quad\left(r_{1}, \theta_{1}\right) \in L_{2},
$$

where

$$
\begin{gathered}
\sigma_{\theta z}=\frac{\mu}{r} \frac{\partial u}{\partial \theta} \\
\sigma_{r z}=\mu \frac{\partial u}{\partial r}
\end{gathered}
$$

$\mu$ denotes the shear modulus of the half-space.

By the method of division (Yuan and Men, 1992; Yuan and Liao, 1996), the half-space is divided into two regions (Figure 2), i.e., the region $\Lambda$ and the region $\Omega$. The region $\Lambda$ is bounded by $L_{2}$ and $C_{1}$, and the region $\Omega$ is the half-space excluding the full circle consisting of $L_{1}$ and $C_{1}$.

The total displacement $u$ can be written as

$$
u=\left\{\begin{array}{ll}
u^{(\mathrm{f})}+u^{(\mathrm{s})} & \left(r_{1}, \theta_{1}\right) \in \Omega \\
u^{(\mathrm{t})} & \left(r_{1}, \theta_{1}\right) \in \Lambda
\end{array},\right.
$$

where $u^{(\mathrm{f})}$ is the free-field wave in the region $\Omega, u^{(\mathrm{s})}$ is the scattered and diffracted wave in the region $\Omega$, and $u^{(\mathrm{t})}$ is the transmitted wave in the region $\Lambda$.

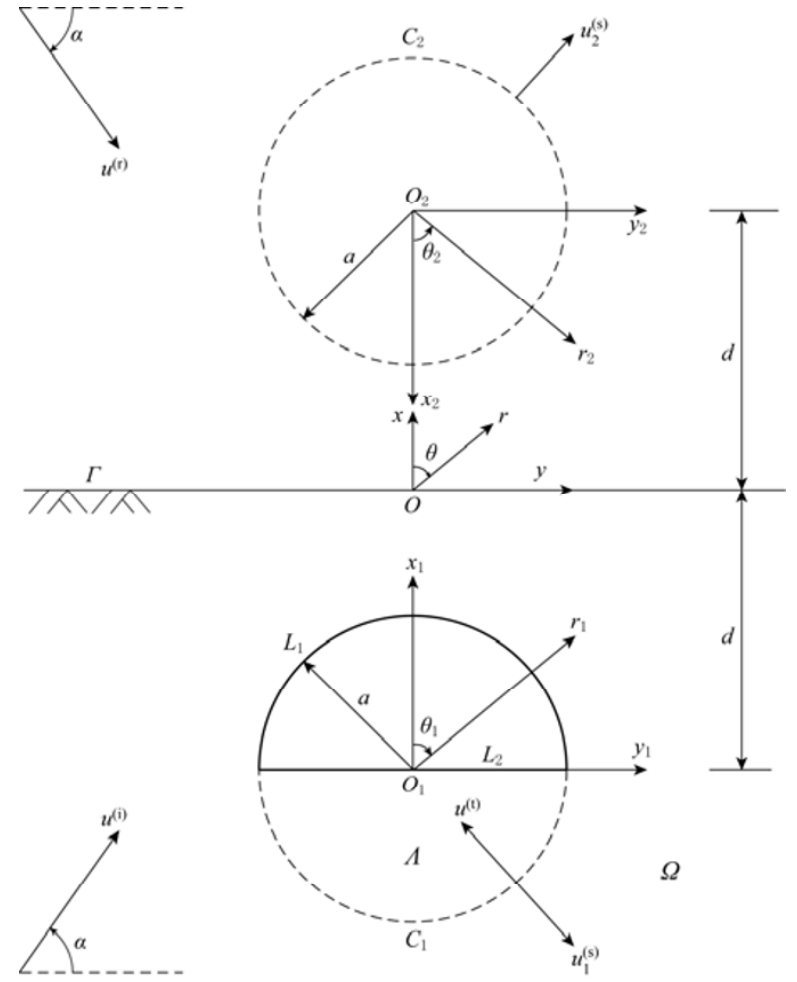

Figure 2 Mathematical model.

More boundary conditions are then introduced due to the division of the half-space, which are the displacement and stress continuity boundary conditions along the boundary $C_{1}$, and can be expressed as

$$
u^{(\mathrm{f})}+u^{(\mathrm{s})}=u^{(\mathrm{t})} \quad\left(r_{1}, \theta_{1}\right) \in C_{1},
$$

and

$$
\sigma_{r_{1} z}^{(\mathrm{f})}+\sigma_{r_{1} z}^{(\mathrm{s})}=\sigma_{r_{1} z}^{(\mathrm{t})} \quad\left(r_{1}, \theta_{1}\right) \in C_{1} .
$$

The free-field response $u^{(\mathrm{f})}$, consisting of the incident wave $u^{(\mathrm{i})}$ and the reflected wave $u^{(\mathrm{r})}$ due to the free surface $\Gamma$ of the half-space (Figure 2), can be expressed as

$$
\begin{gathered}
u^{(\mathrm{f})}\left(r_{1}, \theta_{1}\right)=u^{(\mathrm{i})}+u^{(\mathrm{r})}= \\
u_{0} \sum_{n=0}^{\infty} \mathrm{J}_{n}\left(k r_{1}\right)\left(A_{0, n} \cos n \theta_{1}+B_{0, n} \sin n \theta_{1}\right),
\end{gathered}
$$

where $k=\omega / c_{\beta}$ is wave number, $\mathrm{J}_{n}(x)$ is the Bessel function of the first kind with order $n$, and for $n=0,1,2, \cdots$.

$$
\left\{\begin{array}{l}
A_{0, n}=\varepsilon_{n} \mathrm{i}^{n}\left[\exp (-\mathrm{i} k d \sin \alpha) \cos n\left(\frac{\pi}{2}-\alpha\right)+\exp (\mathrm{i} k d \sin \alpha) \cos n\left(\frac{\pi}{2}+\alpha\right)\right] \\
B_{0, n}=\varepsilon_{n} \mathrm{i}^{n}\left[\exp (-\mathrm{i} k d \sin \alpha) \sin n\left(\frac{\pi}{2}-\alpha\right)+\exp (\mathrm{i} k d \sin \alpha) \sin n\left(\frac{\pi}{2}+\alpha\right)\right]
\end{array}\right.
$$


are coefficients (Liang et al, 2004; Luo, 2003) of the free-field response with $\varepsilon_{0}=1, \varepsilon_{n}=2$ for $n>0$, and i the imaginary unit. The time factor $\exp (-\mathrm{i} \omega t)$ is understood and omitted for all subsequent equations.

Substituting equation (10) into (6) leads to

$$
\sigma_{r_{1} z}^{(\mathrm{f})}\left(r_{1}, \theta_{1}\right)=\frac{\sigma_{0}}{k r_{1}} \sum_{n=0}^{\infty} R_{n}\left(k r_{1}\right)\left(A_{0, n} \cos n \theta_{1}+B_{0, n} \sin n \theta_{1}\right)
$$

where $\sigma_{0}=\mu k u_{0}$ represents the stress amplitude of the incident wave, and

$$
R_{n}\left(k r_{1}\right)=n \mathrm{~J}_{n}\left(k r_{1}\right)-k r_{1} \mathrm{~J}_{n+1}\left(k r_{1}\right) .
$$

For the diffracted wave due to the presence of the cavity, by using the method of separation of variables, the solution satisfying equation (1) and the boundary condition (2) can be written as

$$
u^{(\mathrm{s})}=u_{1}^{(\mathrm{s})}+u_{2}^{(\mathrm{s})},
$$

where

$$
\begin{gathered}
u_{1}^{(\mathrm{s})}\left(r_{1}, \theta_{1}\right)=u_{0} \sum_{n=0}^{\infty} \mathrm{H}_{n}^{(1)}\left(k r_{1}\right)\left(A_{n} \cos n \theta_{1}+B_{n} \sin n \theta_{1}\right), \\
u_{2}^{(\mathrm{s})}\left(r_{2}, \theta_{2}\right)=u_{0} \sum_{n=0}^{\infty} \mathrm{H}_{n}^{(1)}\left(k r_{2}\right)\left(A_{n} \cos n \theta_{2}+B_{n} \sin n \theta_{2}\right),
\end{gathered}
$$

where $A_{n}$ and $B_{n}$ are complex constants to be determined. $\mathrm{H}_{n}^{(1)}(x)$ is the Hankel function of the first kind with order $n$. Substituting equation (15) into (6) leads to

$$
\sigma_{r_{1} z}^{(\mathrm{sl})}\left(r_{1}, \theta_{1}\right)=\frac{\sigma_{0}}{k r_{1}} \sum_{n=0}^{\infty} T_{n}\left(k r_{1}\right)\left(A_{n} \cos n \theta_{1}+B_{n} \sin n \theta_{1}\right)
$$

where

$$
T_{n}\left(k r_{1}\right)=n \mathrm{H}_{n}^{(1)}\left(k r_{1}\right)-k r_{1} H_{n+1}^{(1)}\left(k r_{1}\right) .
$$

By Graf's addition formula (Abramowitz and Stegun, 1972)

$$
\begin{gathered}
\mathrm{H}_{m}^{(1)}\left(k r_{2}\right)\left\{\begin{array}{c}
\cos m \theta_{2} \\
\sin m \theta_{2}
\end{array}\right\}=\sum_{n=0}^{\infty} \mathrm{J}_{n}\left(k r_{1}\right) \frac{\varepsilon_{n}}{2} . \\
\left\{\begin{array}{l}
{\left[\mathrm{H}_{m+n}^{(1)}(2 k d)+(-1)^{n} \mathrm{H}_{m-n}^{(1)}(2 k d)\right] \cos n \theta_{1}} \\
{\left[\mathrm{H}_{m+n}^{(1)}(2 k d)-(-1)^{n} \mathrm{H}_{m-n}^{(1)}(2 k d)\right] \sin n \theta_{1}}
\end{array}\right\} .
\end{gathered}
$$

The diffracted wave $u_{2}^{(\mathrm{s})}$ can be converted into coordinate system $\left(r_{1}, \theta_{1}\right)$.

$$
u_{2}^{(\mathrm{s})}\left(r_{1}, \theta_{1}\right)=u_{0} \sum_{n=0}^{\infty} \mathrm{J}_{n}\left(k r_{1}\right)\left(A_{n}^{*} \cos n \theta_{1}+B_{n}^{*} \sin n \theta_{1}\right),
$$

where

$$
A_{n}^{*}=\sum_{m=0}^{\infty} U_{n m} A_{m} \quad B_{n}^{*}=\sum_{m=0}^{\infty} V_{n m} B_{m}
$$

where

$$
\left\{\begin{array}{l}
U_{n m}=\frac{\varepsilon_{n}}{2}\left[\mathrm{H}_{m+n}^{(1)}(2 k d)+(-1)^{n} \mathrm{H}_{m-n}^{(1)}(2 k d)\right] \\
V_{n m}=\frac{\varepsilon_{n}}{2}\left[\mathrm{H}_{m+n}^{(1)}(2 k d)-(-1)^{n} \mathrm{H}_{m-n}^{(1)}(2 k d)\right] .
\end{array}\right.
$$

Similarly, substituting equation (20) into (6) leads to

$$
\sigma_{r_{1}}^{(\mathrm{s} 2)}\left(r_{1}, \theta_{1}\right)=\frac{\sigma_{0}}{k r_{1}} \sum_{n=0}^{\infty} R_{n}\left(k r_{1}\right)\left(A_{n}^{*} \cos n \theta_{1}+B_{n}^{*} \sin n \theta_{1}\right) .
$$

For the transmitted wave in the region $\Lambda$, by using the method of separation of variables, the solution satisfying equation (1) and the boundary condition (4) can be written as

$$
\begin{gathered}
u^{(\mathrm{t})}\left(r_{1}, \theta_{1}\right)= \\
u_{0} \sum_{n=0}^{\infty} \mathrm{J}_{n}\left(k r_{1}\right)\left(C_{n} \delta_{n}^{(1)} \cos n \theta_{1}+D_{n} \delta_{n}^{(2)} \sin n \theta_{1}\right)
\end{gathered}
$$

where $C_{n}$ and $D_{n}$ are complex coefficients to be determined, and

$$
\left\{\begin{array}{l}
\delta_{n}^{(1)}=1+(-1)^{n}= \begin{cases}0 & \text { when } n \text { odd } \\
2 & \text { when } n \text { even }\end{cases} \\
\delta_{n}^{(2)}=1-(-1)^{n}= \begin{cases}2 & \text { when } n \text { odd } \\
0 & \text { when } n \text { even }\end{cases}
\end{array}\right.
$$

Substituting (24) into (5) leads to

$$
\begin{gathered}
\sigma_{\theta_{1} \mathrm{z}}^{(\mathrm{t})}(r, \theta)=\frac{\mu}{r_{1}} \frac{\partial u^{(\mathrm{t})}}{\partial \theta_{1}}= \\
-\frac{\sigma_{0}}{k r_{1}} \sum_{n=0}^{\infty} n \mathrm{~J}_{n}\left(k r_{1}\right)\left(C_{n} \delta_{n}^{(1)} \sin n \theta_{1}-D_{n} \delta_{n}^{(2)} \cos n \theta_{1}\right)
\end{gathered}
$$

and it is easy to verify the traction-free boundary condition (4), since $\sigma_{\theta_{1 z}}^{(\mathrm{t})}\left(r_{1}, \theta_{1}\right)$ in equation (26) is equal to zero for $\theta_{1}= \pm \pi / 2$ on the boundary $L_{2}$.

Substituting (24) into (6) leads to

$$
\begin{gathered}
\sigma_{\eta_{1} z}^{(\mathrm{t})}\left(r_{1}, \theta_{1}\right)=\mu \frac{\partial u^{(\mathrm{t})}}{\partial r_{1}}= \\
\frac{\sigma_{0}}{k r_{1}} \sum_{n=0}^{\infty} R_{n}\left(k r_{1}\right)\left(C_{n} \delta_{n}^{(1)} \cos n \theta_{1}+D_{n} \delta_{n}^{(2)} \sin n \theta_{1}\right) .
\end{gathered}
$$

So far all the diffracted wave functions are constructed in the coordinate system $\left(r_{1}, \theta_{1}\right)$, and the boundary conditions can be introduced. It should be noted that the traction-free boundary condition (2) has been satisfied both for the free-field wave $u^{(\mathrm{f})}$ and the diffracted wave $u^{(\mathrm{s})}=u_{1}^{(\mathrm{s})}+u_{2}^{(\mathrm{s})}$, and the traction-free boundary equation (4) has been satisfied for transmitted wave $u^{(\mathrm{t})}$. 
Only the boundary conditions (3), (8) and (9) need to be considered, which can be expressed as follows.

1) Traction-free boundary condition along the boundary $L_{1}$

$$
\begin{gathered}
\sigma_{r_{1} z}^{(\mathrm{f})}\left(a, \theta_{1}\right)+\sigma_{r_{1} z}^{(\mathrm{s} 1)}\left(a, \theta_{1}\right)+\sigma_{r_{1} z}^{(\mathrm{s} 2)}\left(a, \theta_{1}\right)=0 \\
-\pi / 2 \leq \theta_{1} \leq \pi / 2
\end{gathered}
$$

2) Displacement and stress continuity conditions along the boundary $C_{1}$

$$
\begin{gathered}
\Phi(\theta)=\left\{\begin{array}{lc}
\sigma_{r_{1} z}^{(\mathrm{f})}\left(a, \theta_{1}\right)+\sigma_{r_{1} z}^{(\mathrm{s} 1)}\left(a, \theta_{1}\right)+\sigma_{r_{1} z}^{(\mathrm{s} 2)}\left(a, \theta_{1}\right) & -\frac{\pi}{2}+2 k \pi \leq \theta_{1} \leq \frac{\pi}{2}+2 k \pi \\
\sigma_{r_{1} z}^{(\mathrm{f})}\left(a, \theta_{1}\right)+\sigma_{r_{1} z}^{(\mathrm{s} 1)}\left(a, \theta_{1}\right)+\sigma_{r_{1} z}^{(\mathrm{s} 2)}\left(a, \theta_{1}\right)-\sigma_{r_{1} z}^{(\mathrm{t})}\left(a, \theta_{1}\right) & \text { otherwise }
\end{array}\right. \\
\Psi(\theta)= \begin{cases}0 & -\frac{\pi}{2}+2 k \pi \leq \theta_{1} \leq \frac{\pi}{2}+2 k \pi \\
u^{(\mathrm{f})}\left(a, \theta_{1}\right)+u_{1}^{(\mathrm{s})}\left(a, \theta_{1}\right)+u_{2}^{(\mathrm{s})}\left(a, \theta_{1}\right)-u^{(\mathrm{t})}\left(a, \theta_{1}\right) & \text { otherwise }\end{cases}
\end{gathered}
$$

where $k=0, \pm 1, \pm 2, \pm 3, \cdots$, then the following two equations are mathematically equivalent to the satisfaction of the equations (28), (29) and (30), respectively:

$$
\begin{array}{ll}
\Phi(\theta)=0 & -\pi \leq \theta \leq \pi, \\
\Psi(\theta)=0 & -\pi \leq \theta \leq \pi .
\end{array}
$$

Next, expand $\Phi(\theta)$ and $\Psi(\theta)$ into Fourier series on $\theta \in[-\pi, \pi]$ and set the coefficients equal to zero, and the following four sets of equations are obtained:

$$
\begin{gathered}
R_{l}(k a) A_{0, l}+T_{l}(k a) A_{l}+R_{l}(k a) A_{l}^{*}= \\
\sum_{n=0}^{\infty} R_{n}(k a) \delta_{n}^{(1)} \lambda_{l n} C_{n},
\end{gathered}
$$

$$
\begin{gathered}
\sum_{n=0}^{\infty}\left[\mathrm{J}_{n}(k a) A_{0, n}+\mathrm{H}_{n}^{(1)}(k a) A_{n}+\mathrm{J}_{n}(k a) A_{n}^{*}\right] \lambda_{l n}= \\
\sum_{n=0}^{\infty} \mathrm{J}_{n}(k a) \delta_{n}^{(1)} \lambda_{l n} C_{n}, \\
R_{l}(k a) B_{0, l}+T_{l}(k a) B_{l}+R_{l}(k a) B_{l}^{*}= \\
\sum_{n=0}^{\infty} R_{n}(k a) \delta_{n}^{(2)} \mu_{l n} D_{n},
\end{gathered}
$$

$$
\begin{gathered}
\sum_{n=0}^{\infty}\left[\mathrm{J}_{n}(k a) B_{0, n}+\mathrm{H}_{n}^{(1)}(k a) B_{n}+\mathrm{J}_{n}(k a) B_{n}^{*}\right] \mu_{l n}= \\
\sum_{n=0}^{\infty} \mathrm{J}_{n}(k a) \delta_{n}^{(2)} \mu_{l n} D_{n},
\end{gathered}
$$

where $l=0,1,2,3, \cdots$,

$$
\begin{aligned}
& \lambda_{l n}=\left\{\begin{array}{ll}
\frac{1}{2} & n=l \\
-\frac{\varepsilon_{l}}{2 \pi} \frac{\sin \frac{n+l}{2} \pi}{n+l}-\frac{\varepsilon_{l}}{2 \pi} \frac{\sin \frac{n-l}{2} \pi}{n-l} & n \neq l
\end{array},\right. \\
& \mu_{l n}=\left\{\begin{array}{ll}
0 & n=l=0 \\
\frac{1}{\pi} \frac{\sin \frac{n+l}{2} \pi}{n+l}-\frac{1}{\pi} \frac{\sin \frac{n-l}{2} \pi}{n-l} & n \neq l \\
\frac{1}{2} & n=l \neq 0
\end{array} .\right.
\end{aligned}
$$

Define the auxiliary functions:
We may solve for $A_{n}$ and $C_{n}$ by combining equations (35) and (36), and for $B_{n}$ and $D_{n}$ by equations (37) and (38). The details are omitted here for the sake of brevity.

\section{Accuracy verification}

To verify the accuracy, we define the following parameters.

1) Dimensionless stress residual along the boundary $L_{1}$

$$
\begin{gathered}
t_{L_{1}}=\frac{\left|\sigma_{r_{1} z}^{(\mathrm{f})}\left(a, \theta_{1}\right)+\sigma_{r_{1} z}^{(\mathrm{s} 1)}\left(a, \theta_{1}\right)+\sigma_{r_{1} z}^{(\mathrm{s} 2)}\left(a, \theta_{1}\right)\right|}{\sigma_{0}} \\
-\pi / 2 \leq \theta \leq \pi / 2
\end{gathered}
$$

2) Dimensionless displacement and stress residuals along the boundary $C_{1}$

$$
u_{C_{1}}=\frac{\left|u^{(\mathrm{f})}\left(a, \theta_{1}\right)+u_{1}^{(\mathrm{s} 1)}\left(a, \theta_{1}\right)+u_{2}^{(\mathrm{s} 2)}\left(a, \theta_{1}\right)-u^{(\mathrm{t})}\left(a, \theta_{1}\right)\right|}{u_{0}}
$$

$\pi / 2 \leq \theta \leq 3 \pi / 2$ 
$t_{C_{1}}=\frac{\left|\sigma_{r_{1} z}^{(\mathrm{f})}\left(a, \theta_{1}\right)+\sigma_{r_{1} z}^{(\mathrm{s} 1)}\left(a, \theta_{1}\right)+\sigma_{r_{1} z}^{(\mathrm{s} 2)}\left(a, \theta_{1}\right)-\sigma_{r_{1} z}^{(\mathrm{t})}\left(a, \theta_{1}\right)\right|}{\sigma_{0}}$

$$
\pi / 2 \leq \theta \leq 3 \pi / 2
$$

These residuals are supposed to be equal to zero theoretically, however, the actual numerical results fluctuate due to cut-off error and Gibbs phenomenon.
Furthermore, dimensionless frequency is defined as the ratio of the diameter of the semi-circular cavity to wavelength of the incident wave as

$$
\eta=\frac{2 a}{\lambda}=\frac{k a}{\pi}=\frac{\omega a}{\pi c_{\beta}} .
$$

Figure 3 shows the displacement and stress residuals along the boundaries $L_{1}$ and $C_{1}$ for buried depth
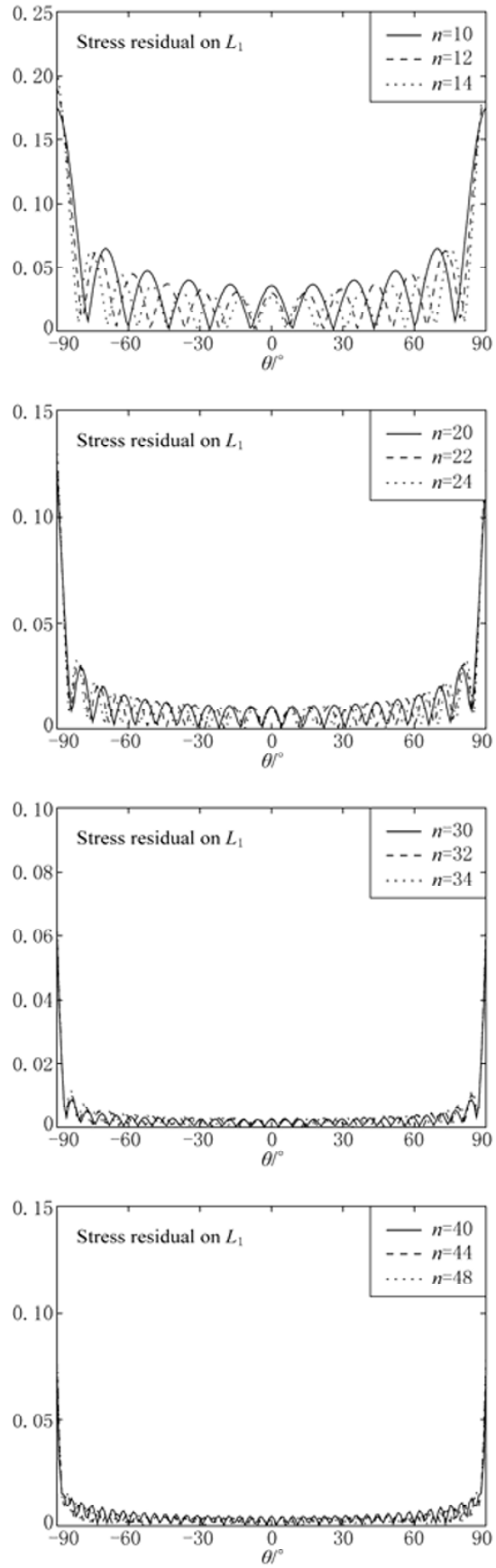
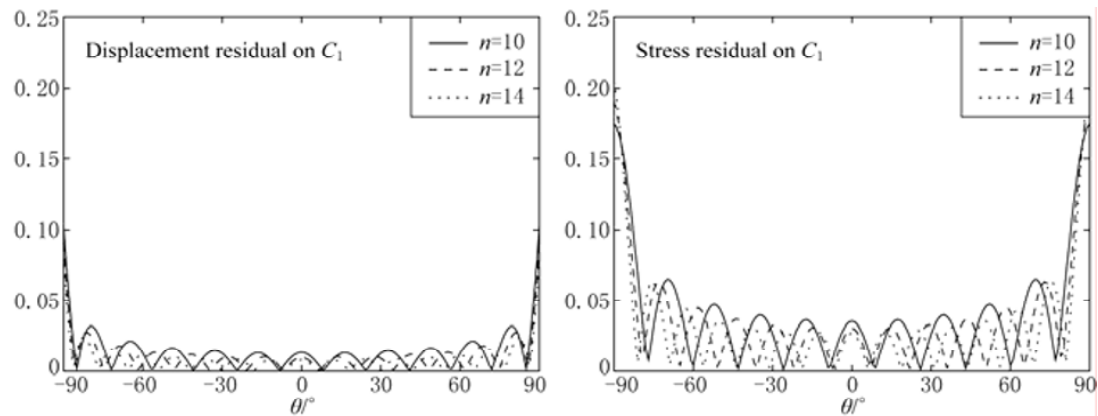

(a)
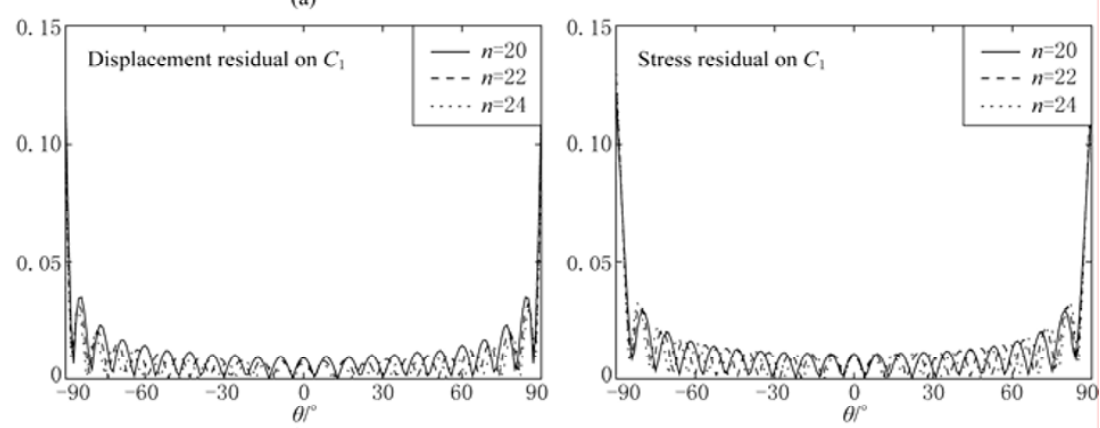

(b)
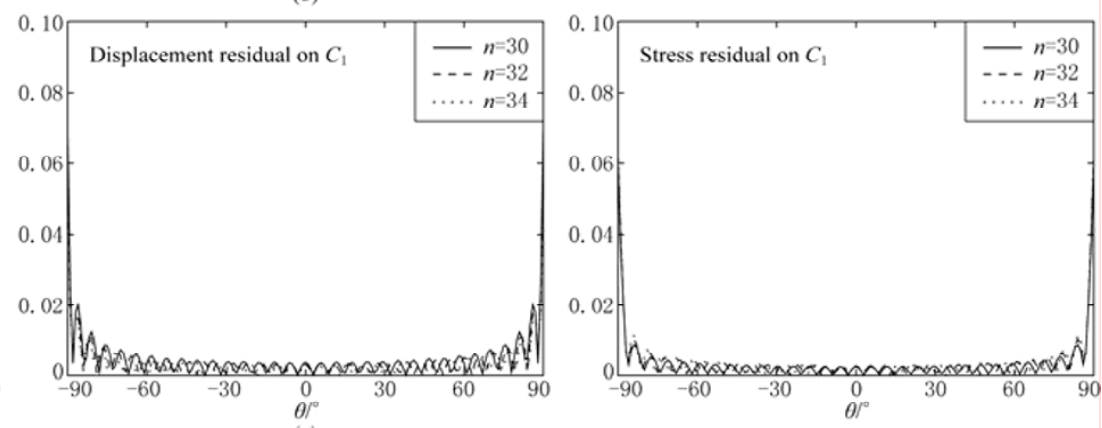

(c)
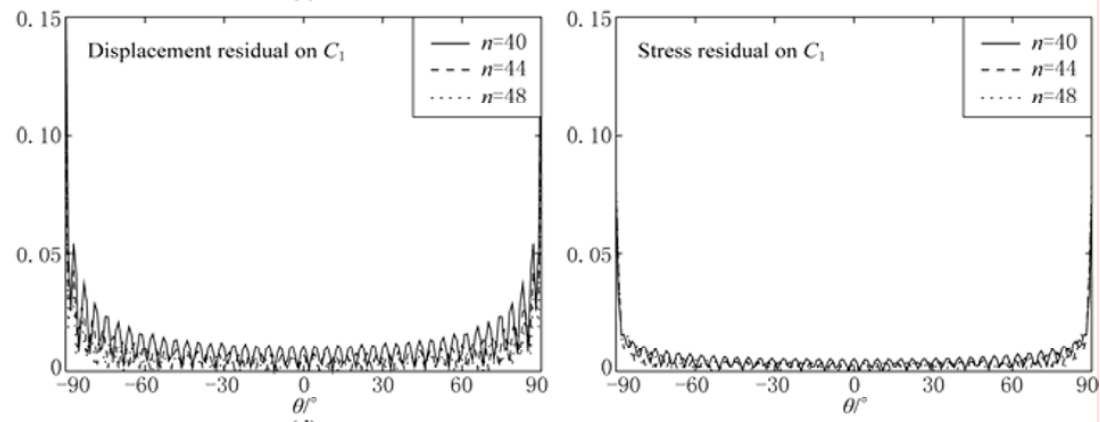

Figure 3 Displacement and stress residuals for buried depth $d / a=2.0$, incident angle $\alpha=90^{\circ}$ in the case of $\eta=1.0$ (a), 3.0 (b), 5.0 (c) and 10.0 (d). 
$d / a=2.0$, incident angle $\alpha=90^{\circ}$, and dimensionless frequency $\eta=1.0,3.0,5.0$, and 10.0, respectively. It can be seen all the displacement and stress residuals approach zero as the truncation order increases, except for those at the rims of the cavity. The large residuals at the rims may be explained by the singularity at the rims and the Gibbs phenomenon due to the discontinuity of the auxiliary functions. Therefore, the displacements and stresses at the rims are not accurate, but the residuals are limited within a local region near the rims. It can be concluded that the truncated series solution presented in this study approaches the true solution when the truncation order is large enough. It can also be seen that the higher the incident frequency is, the larger the truncation order would be.

\section{Numerical results and analysis}

To explore the nature of diffraction of plane $\mathrm{SH}$ waves by a semi-circular cavity in half-space, displacement amplitudes at the surface of half-space and the cavity for different buried depth and different incident
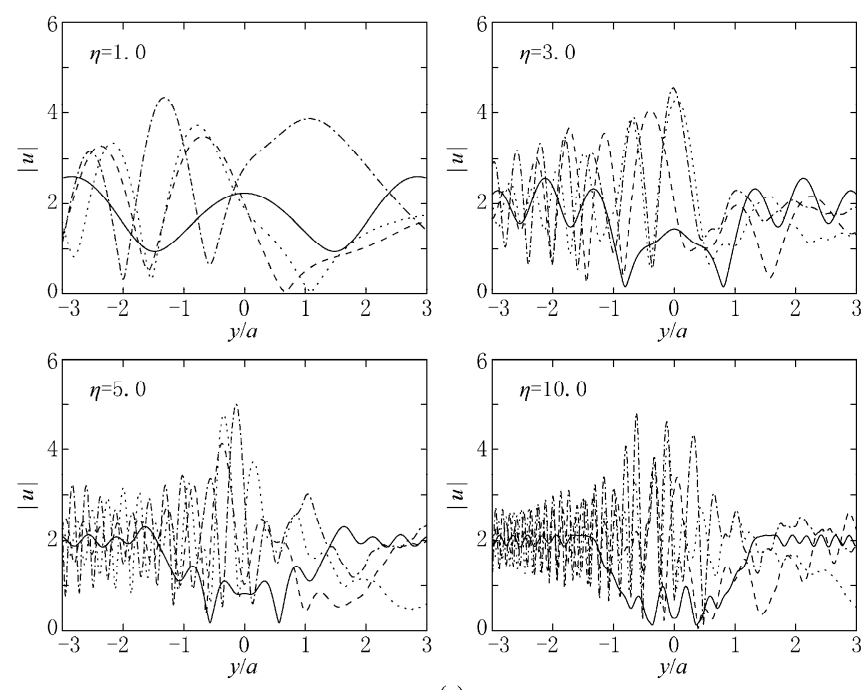

(a)

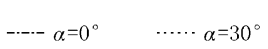

frequency and incident angles are compared with those for a whole-circular cavity in half-space (Lee, 1977).

Figure 4 illustrates the surface displacement amplitudes for buried depth $d / a=2$ and 5, incident angle $\alpha=$ $0^{\circ}, 30^{\circ}, 60^{\circ}$ and $90^{\circ}$, and dimensionless frequency $\eta=$ $1.0,3.0,5.0$, and 10.0, respectively. It is shown from the figure that the surface displacement amplitudes depend strongly on the incident angle and incident frequency; the surface displacement amplitudes reach the maxima for grazing incidence of waves, and the minima for vertical incidence of waves, especially when the incident frequency is high. For vertical incidence of waves the semi-circular cavity behaves an impedance to the incident waves, and the impedance is stronger for the semi-circular cavity than for the whole-circular cavity (Lee, 1977), which is because there is more reflection and less diffraction for the semi-circular cavity (due to the lower flat boundary) than the whole-circular cavity. It can also be seen that the surface displacement amplitudes become smaller for deeper semi-circular cavity, which is similar to that for the whole-circular cavity (Lee, 1977).
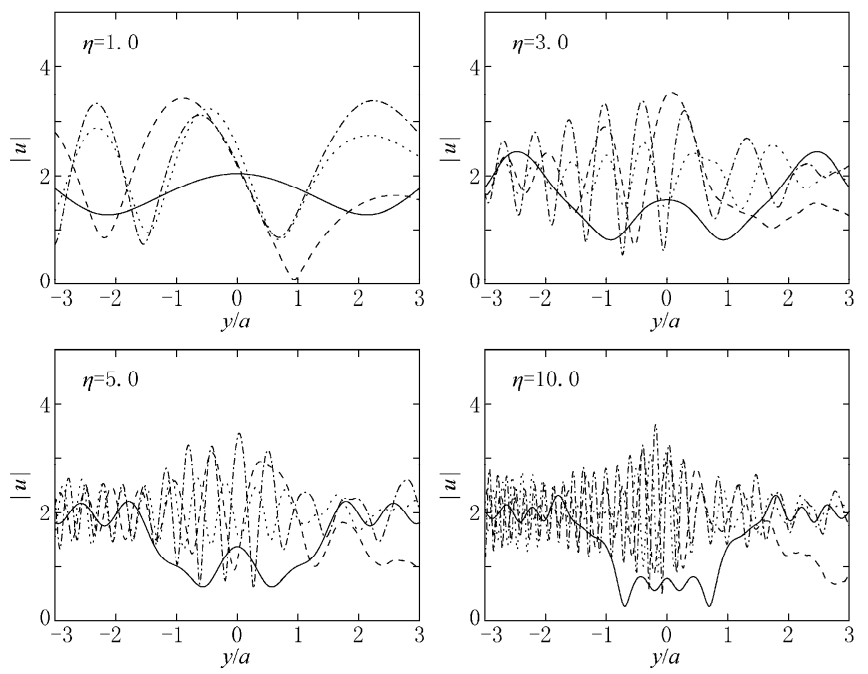

(b)

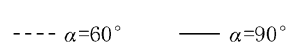

Figure 4 Surface displacement amplitude of half-space for $d / a=2$ (a) and 5 (b).

Figure 5 illustrates the spectral amplification of the displacement amplitude at the top and rims of the semi-circular cavity for buried depth $d / a=2$ and 5, incident angle $\alpha=0^{\circ}$ and $90^{\circ}$, respectively. For comparison, the spectra for the whole-circular cavity are provided as the reference, and the two rims in the figure correspond to $y / a= \pm 1$ within the whole-circular boundary. It is shown that the displacement amplitudes at the top and two rims of the semi-circular cavity depend strongly on the incident angle. For vertical incidence of waves the displacement amplitudes of the semi-circular cavity are slightly smaller than that of the whole-circular cavity, which is due to the more reflection and less diffraction for the semi-circular cavity. For grazing incidence of waves the displacement amplitude at the left rim is much smaller for the semi-circular cavity due to much less re- 
flection than for the whole-circular cavity; however, the displacement amplitude at the right rim is larger for the semi-circular cavity than for the whole-circular cavity for higher incident frequency, which may be caused by more diffraction for the semi-circular cavity than for the whole-cavity.
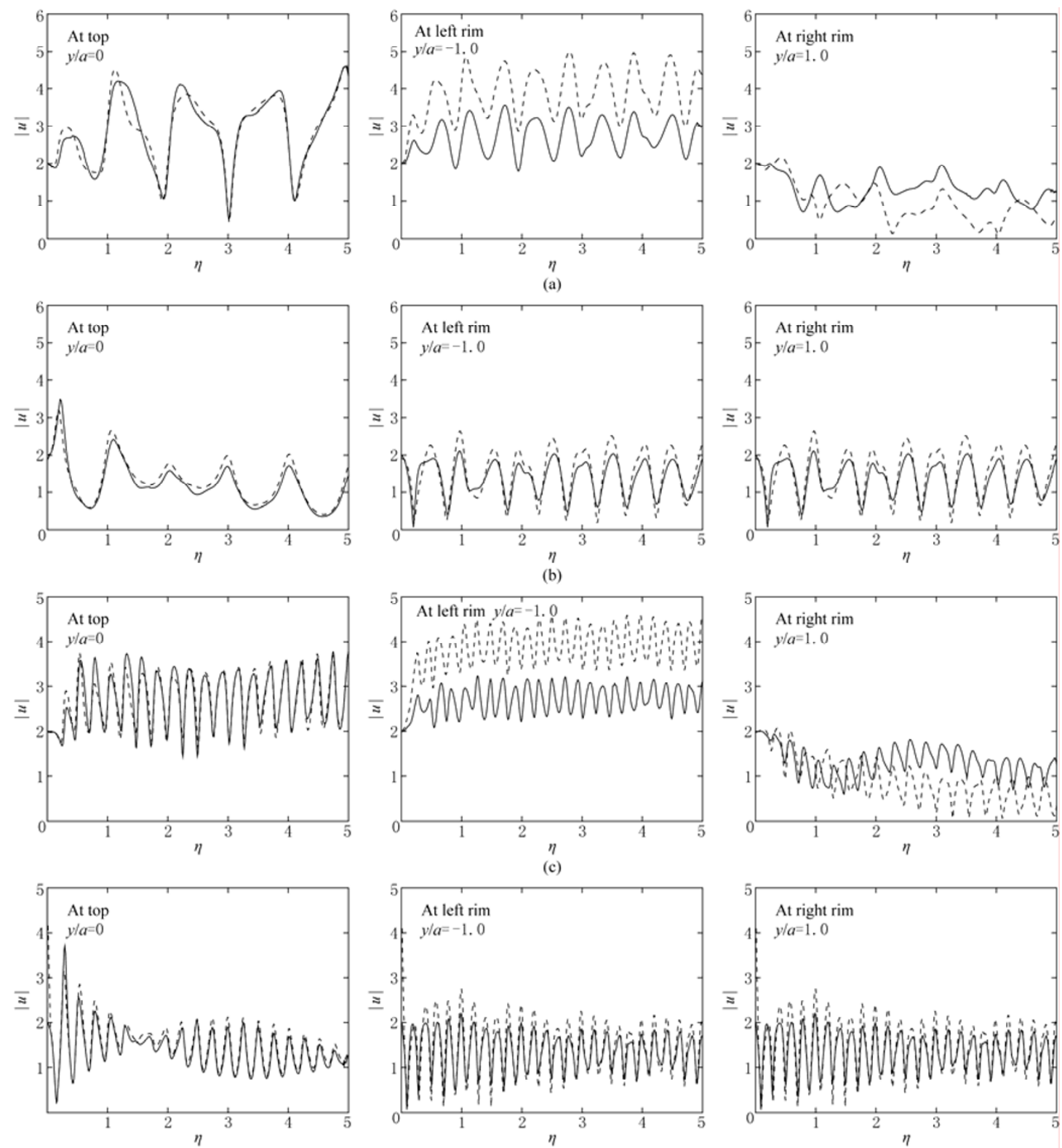

Semi-circle

Figure 5 Surface displacement amplitude spectrum of cavity for different incident angle and buried depth. (a) $\alpha=0^{\circ}$, $d / a=2$; (b) $\alpha=90^{\circ}, d / a=2$; (c) $\alpha=0^{\circ}, d / a=5$; (d) $\alpha=90^{\circ}, d / a=5$.

\section{Conclusions}

A closed-form analytical solution is presented for diffraction of plane SH waves by a semi-circular cavity in half-space by wave function expansion method. The accuracy of the solution is verified by checking the boundary conditions, and numerical results and analysis are performed, and some conclusions are obtained.

Surface displacement amplitudes of half-space depend strongly on the incident angle and incident frequency, and the displacement amplitudes reach the maxima for grazing incidence cases, and the minima for 
vertical incidence cases, especially for high frequency; for vertical incidence of waves the semi-circular cavity behaves like an impedance to the incident waves, and the impedance is stronger for the semi-circular cavity than for the whole-circular cavity.

For vertical incidence of waves the surface displacement amplitudes along the semi-circular cavity are slightly smaller than that of the whole-circular cavity; for grazing incidence of waves the displacement amplitude at the left rim is much smaller for the semi-circular cavity than for the whole-circular cavity, however, the displacement amplitude at the right rim is larger for the semi-circular cavity than for the whole-circular cavity for higher frequency.

Acknowledgements This study is supported by National Natural Science Foundation of China (No. 50978183) and Tianjin Natural Science Foundation (No. 07JCZDJC10100). This research was carried out when the second author studied for his Master's degree in Tianjin University during 2000 to 2003.

\section{References}

Abramowitz M and Stegun I A (1972). Handbook of Mathematical Functions, with Formulas, Graphs, and Mathematical Tables. Dover Publications Inc., New York, 363.

Lee V W (1977). On deformations near circular underground cavity subjected to incident plane SH-waves. In: Wellford C ed. Proceedings of the Application of Computer Methods in Engineering Conference. University of Southern California Press, Los Angeles, USA, 951-962.

Lee V W and Trifunac M D (1979). Response of tunnels to incident SH-waves. J Eng Mech, ASCE 105: 643-659.

Lee V W, Luo H and Liang J (2004). Diffraction of anti-plane SH waves by a semi-circular cylindrical hill with an inside concentric semi-circular tunnel. Earthq Eng Eng Vib 3(2): 249-262.

Liang J, Luo H and Lee V W (2004). Scattering of plane SH waves by a circular-arc hill with a circular tunnel. Acta Seismologica Sinica 17: 549-563.

Liu D and Lin H (2003). Scattering of SH-waves by a shallow buried cylindrical cavity and the ground motion. Explosion and Shock Waves 23(1): 6-12. (in Chinese with English abstract).

Luo H (2003). Scattering of Plane SH Waves by Circular-arc Hill with a Circular Tunnel: An Analytical Solution. Master's Dissertation, Tianjin University, 6-9 (in Chinese with English abstract).

Pao Y-H and Mow C C (1973). Diffraction of Elastic Waves and Dynamics stress Concentrations. Crane, Russak \& Company Inc., New York, 113-125.

Yuan X and Liao Z P (1996). Surface motion of a cylindrical hill of circular-arc cross-section for incident plane SH waves. Soil Dynam Earthq Eng 15: 189-199.

Yuan X and Men F L (1992). Scattering of plane SH waves by a semi-cylindrical hill. Earthq Eng Struct Dynam 21: 1 091-1 098. 